\title{
Design-Thinking, Making, and Innovating: Fresh Tools for the Physician's Toolbox
}

\author{
Albala L, Bober T*, Mallozzi M, Koeneke-Hernandez L, Ku B \\ Sidney Kimmel Medical College, Thomas Jefferson University, Philadelphia, 19107, Philadelphia, United States
}

Copyright $(\mathrm{C} 2018$ by authors, all rights reserved. Authors agree that this article remains permanently open access under the terms of the Creative Commons Attribution License 4.0 International License

\begin{abstract}
Medical school education should foster creativity by enabling students to become 'makers' who prototype and design. Healthcare professionals and students experience pain points on a daily basis, but are not given the tools, training, or opportunity to help solve them in new, potentially better ways. The student physician of the future will learn these skills through collaborative workshops and having dedicated 'innovation time.' This pre-clinical curriculum would incorporate skills centered on (1) Digital Technology and Small Electronics (DTSE), (2) Textiles and Medical Materials (TMM), and (3) Rapid Prototyping Technologies (RPT). Complemented by an on-campus makerspace, students will be able to prototype and iterate on their ideas in a fun and accessible space. Designing and making among and between patients and healthcare professionals would change the current dynamic of medical education, empowering students to solve problems in healthcare even at an early stage in their career. By doing so, they will gain empathy, problem-solving abilities, and communication skills that will extend into clinical practice. Our proposed curriculum will equip medical students with the skills, passion, and curiosity to impact the future of healthcare.
\end{abstract}

Keywords Making, Makerspace, Innovation, Design, Prototyping, Healthcare, Medical Education

\section{Introduction}

Physicians are tinkerers. We dissect, probe, palpate, and auscultate in an attempt to understand how the body works and ultimately use that knowledge to help our patients. Throughout history, physicians developed medical devices to meet the dynamic needs of their patients. For instance, Dr. Rene Laennec wanted to hear lung sounds more clearly so he carved the first stethoscope out of wood by hand. These tools, fundamental to the practice of medicine, were born out of the necessity to improve care; however, modern medical making has been largely outsourced to the medical technology industry. As of the printing of this paper, the authors are all medical students at Sidney Kimmel Medical College of Thomas Jefferson University, the first medical school in the country to incorporate design thinking and medical making into the preclinical curriculum. We want to redesign medical education by enabling medical students to become tinkerers, who prototype and create solutions to problems in healthcare at the earliest stages of their training.

\section{Pain Point of Standard Medical Education}

Preclinical medical education does a wonderful job building the academic foundations of medicine, but provides minimal exposure to the tools, devices, and patient experiences that encompass the practice of medicine. We learn about a wide variety of organ systems, diseases, and procedures - say, the process and clinical relevance of balloon-inflated valvuloplasty in mitral stenosis - without the opportunity to touch, feel, and deconstruct the tools that are being used. Furthermore, disease is taught from every perspective (anatomic, biochemical, pathological, etc.) except that of the patient, whose experiences cannot be summed up in a textbook blurb.

Designing and making with patients would flip the current dynamic of medical education, empowering students to solve problems in healthcare even at this early stage in our career. Quoting Mark Hatch [1], who has written about the Maker Movement, Making allows us to "create and express ourselves to feel whole...things we make are like little pieces of us and seem to embody portions of our soul." Making at the bedside with patients would enable us to reconnect with the human aspect of medicine - the reason why most of us chose this demanding profession. Co-creating not just with patients, but with other members of our multi-disciplinary teams will build 
empathy, nurture problem-solving abilities, and hone communication skills that will extend into clinical practice. Furthermore, these exercises would serve as an extracurricular outlet in a field struggling with epidemic levels of burnout [2-4]. Fueled by diverse engagement and creativity, medical making could produce many dividends for the future of high-quality, patient-centered care.

As we explore the social determinants of health, engaging patients through making could serve as the fulcrum to revolutionize patient care and improve outcomes. Doctors, nurses, and pharmacists may be experts in the clinical aspects of a disease, but only the patient knows how that condition directly impacts their daily lives. What if, rather than prescribing medications for rheumatoid arthritis, doctors worked with patients to identify and solve the problems that go along with that condition - like designing a pill container with wider wells to help someone with limited dexterity? In tailoring solutions to patients, the physician can work together with the patient to guide them in improving their health, rather than running through a checklist of prescribed medications or guidelines for treatment. With patient satisfaction now tied to reimbursements, teaching hospitals would be wise to educate their future physician workforce in the use of techniques that personalize and customize care to meet each patient's needs. The key is to expose young physicians to this human-centered problem solving early in training before they are exposed to, and become entrenched in, algorithm-based medicine.

\section{Solution}

We want to introduce the concept of the daily "hacking" of medical devices to improve healthcare. Modern medicine is outfitted with many tools, but there is no real mechanism to incorporate improvements from innovative users and team members. An ICU nurse may rig a fragile catheter neck with surgical tape to prevent breaks, but how can they communicate this change to a wider community or have a say in redesigning the tool? Valuable clinical insights are useless unless the people who experience them are empowered to be part of the change. In Thursky and Mahemoff [5], they described an ICU where key users (residents and pharmacists) were identified for rapid design of an antibiotic decision support system. When physicians and pharmacists were able to apply their experiences to redesign their own workspace, the resulting solution was better able to meet the needs of their colleagues and patients. Susannah Fox, the Chief Technology Officer for the U.S. Department of Health and Human Services, characterized these types of solutions as "hack(ing) the system in a way that you never would expect...(to) see the future of health care." We agree.

Patients also "hack" their daily lives - inventing individualized solutions to their own problems that could help many more throughout the system. These individuals organize their medications in ways that are the most understandable to them, set up their bathrooms to make it easier to get in and out of the tub, and time their prescription pick-ups with their monthly budgets. This makes sense, as these patients - regardless of their medical or biological literacy - are experts in their own bodies and the milieu of their lives. Whether they realize it or not, they are creating homemade solutions to handle their own complexities, which could apply to help so many others. As clinicians, we need to find ways to integrate our own knowledge into the patient's deep knowledge of their own situations. We are entering an era of "personalized" medicine that will be defined by individuals' genetic makeup or biological markers on their cells. What if the definition of "personalized" was expanded to tailor our solutions to specific aspects of a person's daily life? Integrating making into patient interactions could provide clinicians with deeper understandings of these unique life situations. If we make together, the entire healthcare system and the health outcomes that it produces could be improved.

Medical students have the curiosity, skills, and drive to fuel a community in Making Health. Aspiring physicians have backgrounds in the humanities, business, engineering, arts, and many other areas that could bring fresh perspectives to unsolved problems in healthcare. If medical school could foster a collaborative network to explore, iterate, and prototype new ideas, these experiences could be harnessed productively. In 2015, Jefferson medical students in the Design Track worked with patients in the emergency department to make their own blister pill packs at the bedside; they explored the use of low cost, bicycle pump-powered nebulizers with asthmatic patients in Panama; and they prototyped a drone that delivers health kits to hikers. Imagine the impact that could be made if this diversity of interests was being leveraged at medical schools across the country. We want to help young doctors give voice to their passions using the methodology of the Maker Movement, which empowers everyday people to express themselves through making.

The first step to making something is to generate a forum for individuals to express, generate, and share ideas. Design thinking forces you to interrogate problems with empathy, but requires open dialogue between all involved. You have to think about the experience that anyone who interacts with your idea - a device or process - before you can go about making anything. A growing trend includes healthcare "hackathons," which are team-based innovation competitions that apply the traditional "hacking" mindset to problems in healthcare. No idea is too small or big to be considered or analyzed. Jefferson has established a Health Hack that held on campus each fall. Students can gain firsthand experience in computer programming, 3D printing, as well as burgeoning fields like virtual reality. The beauty of hackathons is that they bring together 
individuals with unique skill sets who may not interact in problem solving. One team may consist of an ICU nurse, a freelance programmer, a businessperson, and a writer who coalesce around an idea. The key to success in the competition is meshing a diverse array of skill sets, as a more unique set of perspectives is more likely to dream up something new and exciting. In healthcare, it is essential that any team member is able to patient care, with the same being true for nurturing innovation in this field.

Once inspired, students and professionals need to have a space and access to materials to make their ideas a reality. Jefferson took this step and established The Vault," the first makerspace established at a United States medical school. The on-campus facility provides access to a bevy of machines - including state-of-the-art 3D printers, virtual reality platforms - as well as essential tools for any project. Students can program a 3D-printed project to print prior to leaving for clinical duties in the morning and arrive to pick up a completed object by the end of their workday. The space provides demonstrations and training on the machines - thereby creating opportunities for students from non-engineering backgrounds to participate and contribute to the programming.

A Medical Maker program would provide a creative and safe space with the tools and support necessary for medical students to gain technical skills and rapidly prototype solutions. Since many academic medical centers are affiliated with, or located in close proximity to, undergraduate institutions, pre-existing resources could be utilized to educate medical students in aspects of engineering, design, business, and other disciplines. Our Medical Maker program will be based on educational sessions in three core technical skill areas: Digital Technology and Small Electronics (DTSE), Textiles and Medical Materials (TMM), and Rapid Prototyping Technologies (RPT). DTSE would expose students to coding principles that would enable them to use microcontroller platforms such as Arduino and Raspberry $\mathrm{Pi}$ and create mobile/web-based applications. Beyond electronic development, this curriculum will introduce students to cutting-edge digital technologies (such as big data/machine learning or blockchain patient records), and inspire applications in healthcare. TMM would introduce skills for working and prototyping with fabric, foam, Legos, or any material that brings an idea to life. This module will also explore the arsenal of tools and machines across healthcare, giving students an opportunity to use tools and practice re-designing them. RPT focuses on Computer Animated Design (CAD) and 3D printing, which could be bolstered by open-access biomedical printing resources such as the NIH 3D Print Exchange. Like any medical research, prototypes and creations advanced to formal testing stages would still require pre-implementation approval through the IRB or FDA. At every step, students will learn more about the environment they will be a part of. The process of collaborating and creating would undoubtedly be a transformative experience for all involved.

Empowering medical students to think and create solutions in healthcare has already paid dividends for students at Thomas Jefferson University. In the spring 2016, a small group of medical students were selected to work with a product design firm in Brooklyn, New York. Each student presented a relevant problem with a proposed solution, and then formed teams to tackle two selected issues. The teams worked on post-exam weekends for six months, developing deeper understanding of their problems through research, building models, and, finally, generating fully-functional prototypes for medical devices. The JeffSolves Program culminated in both teams presenting their projects to investors and interested parties at the newly-renovated Brooklyn Navy Yard. The beauty of this initiative is that it pairs passion and interest with the resources and community to turn ideas into reality. We also learned a great deal about startup culture and processes, meeting with businesspeople and designers at the 10XBeta firm, learning how to pitch to investors, and how to get a new business off the ground. These skills, while not formally taught in most medical schools, are essential to succeed in a variety of healthcare settings. It is also helpful in team management and interrelationships, which guide the workflow of modern healthcare.

JeffSolves produced two patented medical devices that have since become full-fledged startup companies. One medical student team analyzed the inherent friction between 24-hour hospital care and the need for patients to sleep. Simply put, hospital patients cannot get a good night's sleep. As medical students, we often represent the first wave of team members making rounds and commonly hear our patients exclaim, "I' $m$ feeling so much better but I barely got any sleep last night." Poor sleep in the hospital is multifactorial, with noise, light pollution, sounds, and pain all playing a role (among other causes). The team knew that they would need to address one of these aspects and decided on light pollution. To better understand the problem, they sat outside a standard patient room on the fifth floor of Thomas Jefferson University Hospital overnight and recorded each time that the lights were turned on. In all, there were twenty light disruptions that night. Imagine the number of times that a patient wakes up overnight in an Intensive Care Unit room. Using design thinking, they decided to create a device that would help overnight providers perform care tasks without disrupting the patient. When engaging nurses and nursing assistants on the floors, the students found that they needed a light source that would allow them the use of their hands to complete care tasks without turning on the overhead lights in the patient room. Using circadian-friendly LEDs, they engineered Circalights.

Circalights are rechargeable, circadian-friendly lighting pods that wirelessly illuminate care hot spots in patient rooms as a provider approaches. This fall, the devices will 
tested in a small, four-night crossover study with 20 patient rooms on an intermediate-acuity floor at Thomas Jefferson University Hospital. After instructing overnight providers in using the lights, a group of ten patients will be selected to have the pods in their rooms overnight while the other ten will experience routine overnight lighting. The groups will switch after two nights. Patients will fill out short surveys each morning regarding their sleep, their anxiety on the Hospital Anxiety and Depression Scale, and their overall overnight care experience - all of which will be compared before and after crossover. Overnight providers will complete a survey regarding use of the light, where it was placed in the room, if the light provided was sufficient for care tasks, and their overall experience with the device. This essential feedback will guide further design modifications to the devices.

The process of designing and 3D printing for the lighting devices was an exercise in medical making. The team composed of medical students, an aspiring material science engineer, and a business student - was tasked with crafting a form factor that could accommodate a wide variety of care settings and layouts. Using design thinking, we first needed to understand the setup of standard hospital rooms and where Circalights could best be used to help providers without disturbing patient sleep. Employing skills from our JeffSolves experience, we used 3D printing software to adapt our initial ideas for the lights. Researching the best triggering mechanisms for the light we found technology that could be applied to serve other functionalities in the hospital, which expanded the potential utility for the device. In this way, making something addressing one problem enabled students to understand other unmet needs in clinical care. The beauty of this creative process is that it is only limited by the ingenuity and passion of those involved. The company was selected for a startup incubator program at NextFab, a local makerspace in South Philadelphia. Through the RAPID hardware accelerator program, they collaborated with engineers to troubleshoot and iterate versions of the device. Although some team members came with backgrounds in engineering and product design, none of these interactions would have been possible without empowering medical students to explore and tinker through making.

Having experienced third-year clinical clerkships at a large academic medical center, we understand the important of making even more intimately. We see our coworkers "hacking" solutions to problems each day. So many times these people do not realize that they are innovating on an everyday basis. Establishing a framework for making in United States medical schools would help students, but also encourage them to help others to act on their ideas. The Maker Movement is more than building neat things. It represents a culture of empowerment and ingenuity that spans professions. Working with other healthcare providers will help us to understand their perspective on patient care - thereby helping with our background in human centered design thinking, we see countless opportunities to improve processes in the hospital.

Design thinking and fabrication could also be directly incorporated into the medical school curriculum through rotating "clinical immersion" modules during organ-system teaching blocks. Using the Cardiology block as an example, sections of the class would begin with a three-part "design orientation" on design thinking, ethnographic research, and empathy. They would then form small teams to tackle a problem in that field - such as reducing readmissions to the hospital in patients with heart failure. During a 4-week teaching block, students would supplement their educational experience by collaborating with providers and other students, interviewing patients, and, most importantly, making something. The experience would culminate with "innovation rounds" pitching sessions in front of their classmates and mentors. The experience could be easily adapted to promote interdisciplinary collaboration with students of other health professions - thereby approximating the care teams that will form in actual practice. The clinical immersion would allow students to gain deeper insight into different departments and, hopefully, introduce them to problems that they will pursue throughout medical school and beyond.

\section{Conclusions}

The healthcare start-up culture is blooming worldwide as individuals aim to tackle the challenges of our current system. By empowering patients and providers who have ideas for solutions, we could aim for more equitable and effective care. It is important for medical professionals to be active participants in the coming healthcare evolutions, so that we may steer such changes in a manner that upholds the various principles of patient-centered care.

We are confident that today's medical student will embrace the tinkering legacy in medicine and develop tools that will revolutionize healthcare delivery. A Medical Maker curriculum will foster deep empathy, clarity of purpose, and inter-professional collaboration - core competencies for a 21st century physician. Medical makerspaces lend a creative launching pad to start thinking outside the box and start identifying solutions to everyday challenges in healthcare.

\section{Acknowledgements}

We would like to thank Dr. Bon $\mathrm{Ku}$, who inspired us and mentored us through a real-life execution of a design curriculum within a medical school. We are also very grateful for the individuals at Thomas Jefferson University that support us directly and indirectly, through the design curriculum. 


\section{REFERENCES}

[1] Hatch, Mark, The Maker Movement Manifesto, McGraw Hill Education, USA, 2014.

[2] Hojat, M., Gonnella, J. S., Nasca, T. J., Mangione, S., Vergare, M., \& Magee, M. Physician empathy: definition, components, measurement, and relationship to gender and specialty. American Journal of Psychiatry, 159(9), 1563-1569, 2002.

[3] Hojat, M., Mangione, S., Nasca, T. J., Rattner, S., Erdmann, J. B., Gonnella, J. S., \& Magee, M. An empirical study of decline in empathy in medical school. Medical education, 38(9), 934-941, 2004.

[4] Shanafelt, T. D., Boone, S., Tan, L., Dyrbye, L. N., Sotile, W., Satele, D., Oreskovich, M. R. Burnout and satisfaction with work-life balance among US physicians relative to the general US population. Archives of internal medicine, 172(18), 1377-1385, 2012.

[5] Thursky, K. A., \& Mahemoff, M. User-centered design techniques for a computerised antibiotic decision support system in an intensive care unit. International journal of medical informatics, 76(10), 760-768, 2007. 